\title{
Rotational Symmetry Breaking in a Trigonal Superconductor Nb-doped $\mathrm{Bi}_{2} \mathrm{Se}_{3}$
}

\author{
Tomoya Asaba, ${ }^{1}$ B. J. Lawson, ${ }^{1}$ Colin Tinsman, ${ }^{1}$ Lu Chen, ${ }^{1}$ Paul Corbae, ${ }^{1}$ Gang Li, ${ }^{1}$ Y. Qiu, ${ }^{2}$ \\ Y. S. Hor, ${ }^{2, *}$ Liang $\mathrm{Fu}^{3}$ and $\mathrm{Lu} \mathrm{Li}^{1, \uparrow}$ \\ ${ }^{1}$ Department of Physics, University of Michigan, Ann Arbor, Michigan 48109, USA \\ ${ }^{2}$ Department of Physics, Missouri University of Science and Technology, Rolla, Missouri 65409, USA \\ ${ }^{3}$ Department of Physics, Massachusetts Institute of Technology, Cambridge, Massachusetts 02309, USA \\ (Received 24 March 2016; revised manuscript received 10 November 2016; published 27 January 2017)
}

\begin{abstract}
The search for unconventional superconductivity has been focused on materials with strong spin-orbit coupling and unique crystal lattices. Doped bismuth selenide $\left(\mathrm{Bi}_{2} \mathrm{Se}_{3}\right)$ is a strong candidate, given the topological insulator nature of the parent compound and its triangular lattice. The coupling between the physical properties in the superconducting state and its underlying crystal symmetry is a crucial test for unconventional superconductivity. In this paper, we report direct evidence that the superconducting magnetic response couples strongly to the underlying trigonal crystal symmetry in the recently discovered superconductor with trigonal crystal structure, niobium $(\mathrm{Nb})$-doped $\mathrm{Bi}_{2} \mathrm{Se}_{3}$. As a result, the in-plane magnetic torque signal vanishes every $60^{\circ}$. More importantly, the superconducting hysteresis loop amplitude is enhanced along one preferred direction, spontaneously breaking the rotational symmetry. This observation indicates the presence of nematic order in the superconducting ground state of $\mathrm{Nb}$-doped $\mathrm{Bi}_{2} \mathrm{Se}_{3}$.
\end{abstract}

DOI: 10.1103/PhysRevX.7.011009

Subject Areas: Superconductivity, Topological Insulators

\section{INTRODUCTION}

Unconventional superconductors are characterized by superconducting order parameters that are noninvariant under crystal symmetry operations. When the order parameter is a single component, this noninvariance is manifested solely in the phase of the superconducting wave function and can only be detected by phase-sensitive measurements. On the other hand, when the order parameter is multicomponent, the magnitude of the superconducting gap can be different along symmetry-related crystallographic directions. The gap anisotropy leads directly to a thermodynamic property of the superconducting state that spontaneously breaks the crystal rotational symmetry of the normal state. Prior to this work, no direct thermodynamic signature of rotational symmetry breaking due to superconductivity has been observed in any crystal.

Bismuth selenide $\left(\mathrm{Bi}_{2} \mathrm{Se}_{3}\right)$ is one of the most promising materials to explore for unconventional superconductivity. Strong spin-orbit coupling in the triangular lattice produces a topologically insulating ground state [1,2]. Doping with a metallic element such as copper $(\mathrm{Cu})$, strontium $(\mathrm{Sr})$, or niobium $(\mathrm{Nb})$ makes it superconducting [3-11]. We report

\footnotetext{
*Corresponding author. yhor@mst.edu

Corresponding author. luli@umich.edu

Published by the American Physical Society under the terms of the Creative Commons Attribution 3.0 License. Further distribution of this work must maintain attribution to the author(s) and the published article's title, journal citation, and DOI.
}

here the first direct observation of rotational symmetry breaking in the superconducting state of $\mathrm{Nb}$-doped $\mathrm{Bi}_{2} \mathrm{Se}_{3}$ [12], a new member of the superconducting doped topological insulators. Possible odd-parity pairing symmetries in doped $\mathrm{Bi}_{2} \mathrm{Se}_{3}$, favored by strong spin-orbit interactions, have been theoretically proposed and classified according to the representations of the $D_{3 d}$ point group [5]. Among them, only the odd-parity pairing in the two-dimensional $E_{u}$ representation gives rise to a nematic superconductor with broken rotational symmetry [13]. A recent nuclear magnetic resonance experiment on $\mathrm{Cu}_{x} \mathrm{Bi}_{2} \mathrm{Se}_{3}$ reveals an in-plane anisotropy in the spin susceptibility of the superconducting state [14], providing spectroscopic evidence for the $E_{u}$ pairing. An electrical transport study on Sr-doped $\mathrm{Bi}_{2} \mathrm{Se}_{3}$ reveals the anisotropy of upper critical field $H_{c 2}$ [15]. Heat capacity measurements also show the anisotropy of $H_{c 2}$ [16]. Yet, there is not clear evidence showing the anisotropy in the superconducting properties deep in the superconducting state at $H$ much smaller than $H_{c 2}$. To solve this problem, we chose a unique method, torque magnetometry, to resolve the anisotropy of the superconducting critical current in these materials.

Our torque magnetometry approach is a new and powerful tool to investigate the nematicity. In general, rotational symmetry-breaking measurements require noncontact methods such as heat capacity, magnetization, or NMR since contact itself may generate a preferred axis. However, the combination of field rotation and thermal measurements or NMR requires special effort, while torque magnetometry can be performed in a rather standard and direct setup. In addition, we focus on the symmetry of the hysteresis loop as well as magnetic susceptibility in the superconducting 
state. As far as we know, this is the first time torque magnetometry has been used to investigate nematic order in the superconducting state. By contrast, torque magnetometry has been used to detect nematicity only in the normal state in superconductors $\mathrm{URu}_{2} \mathrm{Si}_{2}$ or $\mathrm{BaFe}_{2}\left(\mathrm{As}_{1-x} \mathrm{P}_{x}\right)_{2}$ $[17,18]$. Thus, our new approach paves the way for further studies on nematicity in the superconducting ground state.

More importantly, observing nematicity in the superconducting state itself is an exciting result. As mentioned above, observing nematicity in $\mathrm{URu}_{2} \mathrm{Si}_{2}$ and $\mathrm{BaFe}_{2}\left(\mathrm{As}_{1-x} \mathrm{P}_{x}\right)_{2}$ has had a huge impact on the field since it significantly helps us understand the unconventional and complicated behaviors of those superconductors. Similarly, rotational symmetry breaking in the superconducting state suggests spin-triplet pairing and topological superconductivity. Thus, our approach and results greatly impact future research on topological superconductivity.

\section{EXPERIMENTAL DETAILS}

The detailed growth method of Nb-doped $\mathrm{Bi}_{2} \mathrm{Se}_{3}$ single crystals is described in Ref. [12]. Crystal orientation is determined by $\mathrm{x}$-ray diffraction on single crystals. The x-ray diffraction pattern is known to be almost identical to $\mathrm{Bi}_{2} \mathrm{Se}_{3}$ [19]. The crystal orientation was determined from a $2 \theta=43.7^{\circ}$ peak corresponding to the (110) plane.

We used torque magnetometry to measure the superconducting hysteresis loop and magnetization of $\mathrm{Nb}$-doped $\mathrm{Bi}_{2} \mathrm{Se}_{3}$. Since the free energy with respect to the external field $H$ is given by $\mathcal{F}=\mu_{0} V \vec{M} \times \vec{H}$, magnetic torque is given by the derivative of $\mathcal{F}$ with respect to the magnetic field tilt angle $\phi: \vec{\tau}=\mu_{0} V \vec{M} \times \vec{H}$. Here, $V$ is the volume of the sample, $\vec{H}$ is the external magnetic field, and $\vec{M}$ is the magnetization of the sample. Torque magnetometry is thus a thermodynamic probe that measures the free energy directly.

The torque is measured by mounting the sample standing on its edge in order to keep the external field in the sample's $a b$ plane [see Fig. 1(a)]. We then rotate the cantilever. This measures the in-plane anisotropy of the sample's magnetic properties in the superconducting state. In the general case, the dominating term of paramagnetic torque is a periodic function of double the azimuthal angle $\phi$ :

$\tau_{2 \phi}=\frac{1}{2} \mu_{0} V M H^{2}\left\{\left(\chi_{a a}-\chi_{b b}\right) \sin 2 \phi-2 \chi_{a b} \cos 2 \phi\right\}$.

Here, $M_{i}=\Sigma_{j} \chi_{i j} H_{j}$. In a system with high symmetry (e.g., tetragonal or hexagonal), $\tau_{2 \phi}$ becomes 0 because $\chi_{a a}=\chi_{b b}$ and $\chi_{a b}=0$. In such a case, the leading torque term becomes

$$
\tau_{2 n \phi}=A_{2 n \phi} \sin 2 n\left(\phi-\phi_{0}\right),
$$

where $n$ is determined by the crystalline symmetry: e.g., $n=2$ for the tetragonal lattice or $n=3$ for the hexagonal
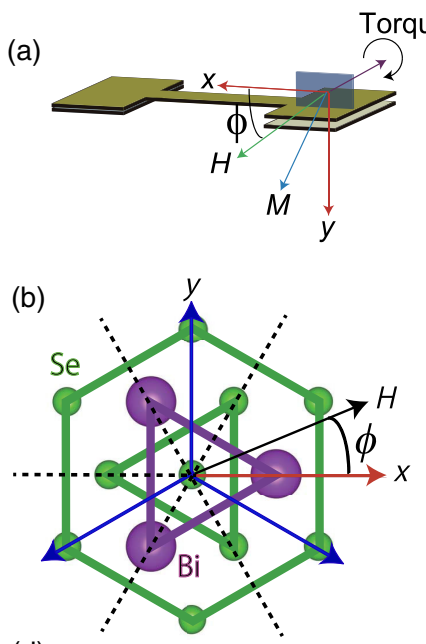

(c)

(d)

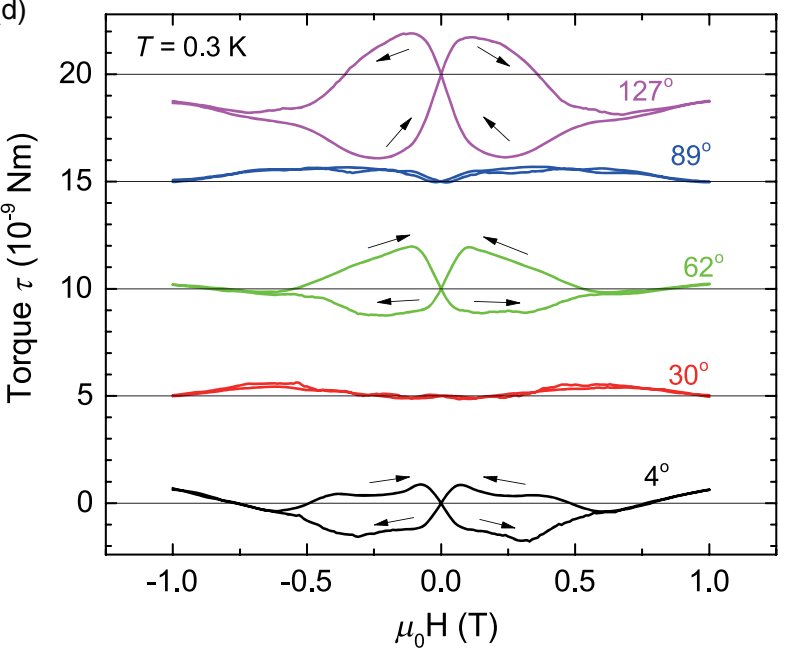

FIG. 1. Experimental setup, sample orientation, and torque curves of $\mathrm{Nb}_{x} \mathrm{Bi}_{2} \mathrm{Se}_{3}$. (a) Schematic sketch of torque magnetometry under in-plane field rotation. The magnetic field is applied in plane. The azimuthal angle $\phi$ is defined as the angle between the external magnetic field and the cantilever arm ( $x$ axis). Torque $\vec{\tau}=\mu_{0} V \vec{M} \times \vec{H}$ is tracked by measuring the capacitance between the cantilever and the gold film beneath it. (b) Crystal structure of $\mathrm{Nb}_{x} \mathrm{Bi}_{2} \mathrm{Se}_{3}$ viewed down the crystalline $\hat{c}$ axis. The dashed lines are the mirror planes of the crystal, and the blue arrows are the crystal axes. These crystal axes are shown to be the directions where the torque loop vanishes. (c) Meissner effect from the sample. The volume magnetic susceptibility reaches close to -1 , indicating a fully superconducting volume. (d) Selected torque curves at $0.3 \mathrm{~K}$ with external magnetic fields between -1 and $1 \mathrm{~T}$. The magnitude of the hysteresis loop is at a maximum at around $120^{\circ}$ and is nearly zero at $30^{\circ}$ and $90^{\circ}$.

lattice. For tetragonal $\mathrm{URu}_{2} \mathrm{Si}_{2}$ or $\mathrm{BaFe}_{2}\left(\mathrm{As}_{1-x} \mathrm{P}_{x}\right)_{2}$ $[17,18], \sin 4 \phi$ is dominant at high temperatures, while $\sin 2 \phi$ becomes pronounced at low temperatures, indicating the emergence of nematic order at low temperatures. In our case, Fig. 1(b) shows the crystal structure of $\mathrm{Nb}$-doped $\mathrm{Bi}_{2} \mathrm{Se}_{3}$ looking down the hexagonal axis. As shown in the figure, the external field is in the hexagonal plane. Thus, in a paramagnetic normal state, a $\sin 6 \phi$ torque response is 
(a)

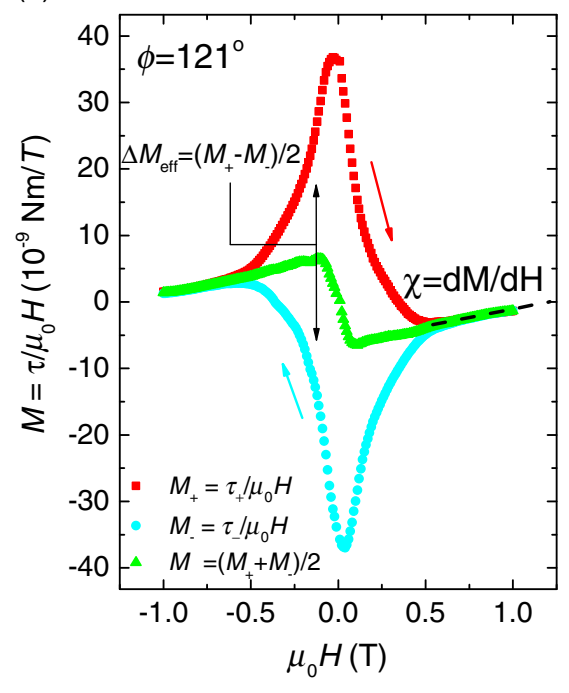

(b)

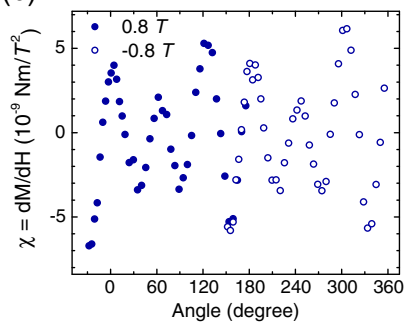

(d)

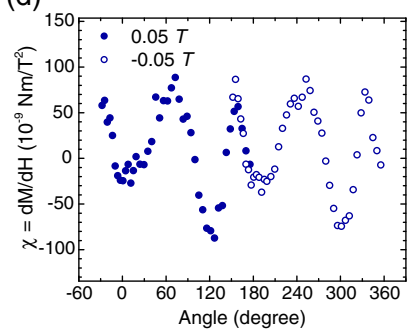

Normal state

(c)

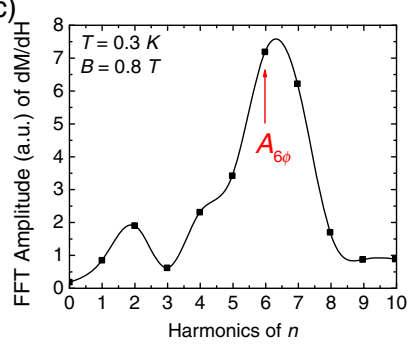

(e)

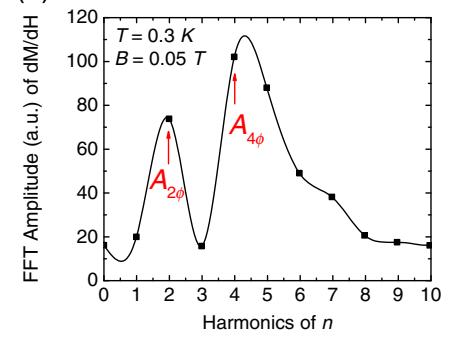

Mixed state

FIG. 2. The angular dependence of susceptibility of $\mathrm{Nb}_{x} \mathrm{Bi}_{2} \mathrm{Se}_{3}$ in a normal state and superconducting state. (a) Examples of the hysteretic $M-H$ curves demonstrate the definition of $M, \Delta M$, and $\chi$. Note that $M_{ \pm}=\tau_{ \pm} / \mu_{0} H, M=\left(M_{+}+M_{-}\right) / 2$, $\Delta M=\left(M_{+}-M_{-}\right) / 2$, and $\chi=d M / \mu_{0} d H$, where $\tau_{+}$is the torque signal from the up-sweep of the magnetic field, and $\tau_{-}$is the torque signal from the down sweep of the magnetic field. (b) Angular dependence of effective susceptibility $\chi$ in a normal state at $0.3 \mathrm{~K}$. A magnetic field of $0.8 \mathrm{~T}$ was applied to suppress the superconductivity. The angle $\phi$ is defined as the tilt angle between the positive $x$ axis and the magnetic field direction. Reversing the sign of the magnetic field is equivalent to rotating the cantilever $180^{\circ}$. We thus used the negative field values to complete the $360^{\circ}$ angular dependence (open circles). It is clearly shown that $\sin 6 \phi$ is dominant. This indicates that the crystal has hexagonal symmetry. (c) The FFT plot of the data shown in (b). Here, $A_{6 \phi}$ is dominant, while $A_{2 \phi}$ and $A_{4 \phi}$ are very small. (d) Angular dependence of effective susceptibility $\chi$ in a superconducting state at $0.3 \mathrm{~K}$. The crystal symmetry is lower than the normal state, resulting in a lower $n$ periodic function of $\phi$. (e) FFT plot of the data shown in (d). Note that $A_{2 \phi}$ and $A_{4 \phi}$ are dominant, while $A_{6 \phi}$ is very small. The contrast between the pattern in the normal state and the superconducting state demonstrates the breaking of the rotational symmetry in the superconducting state.

expected from the crystal structure. In this case, anisotropy in the magnetic susceptibility would bring the magnetic torque to zero along the in-plane mirror lines and the normal axis of the mirror lines of the crystal. The azimuthal angle $\phi$ is the angle between the external magnetic field and the $x$ axis defined by the cantilever arm. Based on the x-ray diffraction pattern of this particular sample, we find that $\phi=0^{\circ}, 60^{\circ}$, and $120^{\circ}$ correspond to the in-plane mirror line of the crystal, as shown by the dashed lines in Fig. 1(b). In the same crystal structure diagram, the blue axes are the in-plane crystal axes, which are $30^{\circ}$ away from the mirror lines.

The $\mathrm{Nb}$-doped $\mathrm{Bi}_{2} \mathrm{Se}_{3}$ sample used in our experiment has a superconducting volume close to $100 \%$, as shown by the volume magnetic susceptibility which approaches -1 in the zero-field cooled run [see Fig. 1(c)]. This is much higher than that of $\mathrm{Cu}$-doped $\mathrm{Bi}_{2} \mathrm{Se}_{3}[8,9]$.

The measured torque shows a strong superconducting signal. Figure 1(d) shows some examples of the magnetic torque on the cantilever from the sample. The torque $\tau$ is plotted as a function of the external magnetic field at temperate $T=0.3 \mathrm{~K}$. We swept the field up and down from $-1 \mathrm{~T}$ to $1 \mathrm{~T}$ to measure the entire superconducting hysteresis loop. Arrows along the curve show the field sweep direction. The $\tau-H$ loop is a signature of the strong flux pinning characteristic of type-II superconductors. The pinned flux lines form a vortex solid, and the flux density inside the superconductor always resists the change in the applied magnetic field. A simple analysis based on the Bean model shows that the hysteresis of the magnetization gives a direct measurement of the superconducting critical current density in the mixed state of type-II superconductors (see Supplemental Material Ref. [20]).

To probe the symmetry of the superconducting sample, we focused on both the symmetry of magnetic susceptibility and the hysteresis loop. We simply define $M_{ \pm}=\tau_{ \pm} / \mu_{0} H$, $M=\left(M_{+}+M_{-}\right) / 2$, and $\Delta M_{\text {eff }}=\left(M_{+}-M_{-}\right) / 2$, as well as the effective magnetic susceptibility $\chi=\left[d M /\left(\mu_{0} d H\right)\right]$, where $\tau_{+}\left(\tau_{-}\right)$is the sample's magnetic torque from the $H$ increasing (decreasing) sweeps, respectively [Fig. 2(a)]. Since the critical field $H_{c 2}$ is about $0.6 \mathrm{~T}$, above $0.6 \mathrm{~T}$ the sample enters the normal state and $\tau_{+}$and $\tau_{-}$(also $M_{+}$ and $M_{-}$) overlap with each other. Below the critical field, $M=\left(M_{+}+M_{-}\right) / 2$ is the average magnetization from the mixed state. From the Bean model, supercurrents induced by field sweeping up and field sweeping down contribute 
(a)

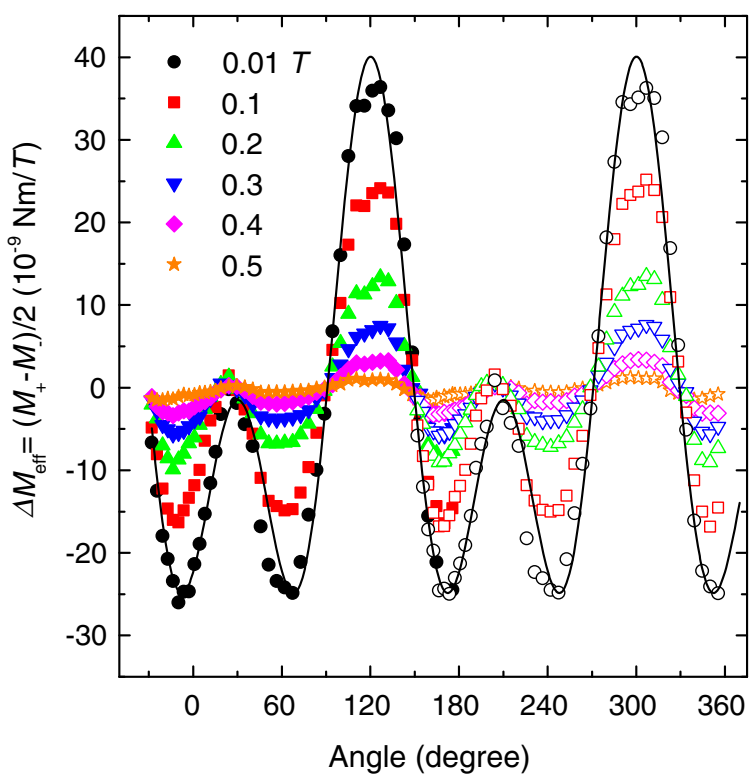

(b)

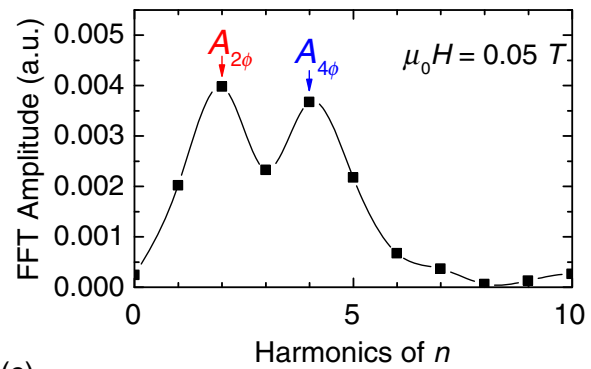

(c)

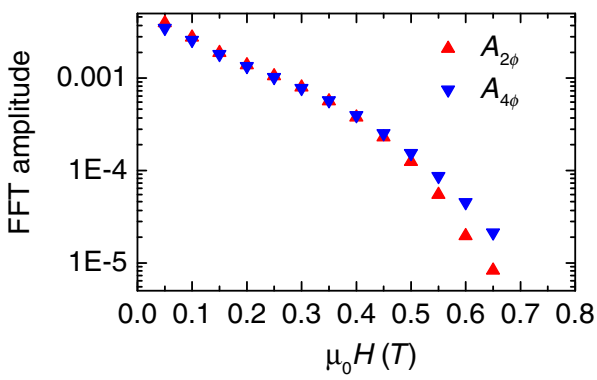

FIG. 3. The angular dependence of spontaneous effective magnetization. (a) The angular dependence of spontaneous effective magnetization $\Delta M-\phi$. The data were taken at $0.3 \mathrm{~K}$ at a few selected $H$ fields. Data taken from the positive field sweep are plotted as filled symbols, and data from the negative field sweep are plotted as open symbols. The solid lines show $f(\phi)=2 A_{2 \phi} \sin \left(\phi-30^{\circ}\right) \cos 3 \phi$. (b) FFT of $\Delta M-\phi$ at $\mu_{0} H=0.05 \mathrm{~T}$. The first peak, $A_{2 \phi}$, is the amplitude of the nematic order term. The second peak, $A_{4 \phi}$, represents the $\sin 4 \phi$ term originating from the product fit function $f(\phi)=2 A_{2 \phi} \sin \left(\phi-30^{\circ}\right) \cos 3 \phi$. (c) The magnetic field dependence of the FFT amplitudes $A_{2 \phi}$ and $A_{4 \phi}$ in the superconducting state of $\mathrm{Nb}$-doped $\mathrm{Bi}_{2} \mathrm{Se}_{3}$. The FFT amplitude is plotted in logarithmic scale for clarity. Above $0.6 \mathrm{~T}$, the superconducting hysteresis loop quickly vanishes as $H$ approaches the upper critical field.

equally, so $M=\left(M_{+}+M_{-}\right) / 2$ corresponds to the intrinsic magnetic susceptibility of the sample. The sample was measured at $T=0.3 \mathrm{~K}$ over an angular range of $200^{\circ}$. Reversing the sign of the magnetic field is equivalent to rotating the cantilever $180^{\circ}$, so we extend the negative field sweep data to complete the $360^{\circ}$ angular dependence.

We first check the crystalline symmetry in the normal state at high fields. In the normal state, the effective magnetic susceptibility $\chi$ shows a periodic pattern with a period of $60^{\circ}$. Note that, while the actual anisotropic susceptibility is constant, the effective susceptibility $\chi=\left(d M /\left[\mu_{0} d H\right]\right)$ is multiplied by the angular factor $\sin 2 n \phi$, resulting in zero or negative $\chi$. As stated above in Eq. (2), this $60^{\circ}$ period is expected from the hexagonal lattice of the samples. Figure 2(b) shows the angular dependence of the susceptibility $\chi$ at $0.8 \mathrm{~T}$, well above the critical field. The normal state torque clearly follows a periodic function of $6 \phi$. The fast Fourier transformation (FFT) of the data in Fig. 2(b) is shown in Fig. 2(c). As shown, the Fourier component of $6 \phi$ is dominant in the normal state. If the sample symmetry is broken, the contribution from the $2 \phi$ component should be orders of magnitude larger. Therefore, a small $2 \phi$ component would be from the higher-order term of the geometric factors. Thus, we conclude that the sample is hexagonal in the normal state.
Now, we focus on the symmetry of the superconducting state. In a superconducting state, the torque curve cannot be explained by the formula for a paramagnetic system. However, in analogy with the torque response of a hexagonal ferromagnetic material, $\tau=A_{6 \phi} \sin 6 \phi$, the torque curve from the hexagonal superconducting material should follow the $\sin 6 \phi$ dependence as well.

In contrast, the angular dependence of our data clearly breaks the $\sin 6 \phi$ pattern. Figure 2(d) shows the angular dependence of the magnetic susceptibility at $0.05 \mathrm{~T}$, well below the critical field. It is clearly shown that the angular dependence of the susceptibility is different from the normal state. Figure 2(e) shows the FFT of the data in Fig. 2(d). Notice that the $2 \phi$ and $4 \phi$ components are dominant rather than the vanished $6 \phi$ component. This implies that the superconducting state breaks the rotational symmetry required by the crystalline lattice.

Finally, the symmetry breaking in the superconducting state is even more pronounced in the hysteresis loop. Figure 3(a) shows the magnitude of the hysteresis of the magnetization $\Delta M_{\text {eff }}=\left(M_{+}-M_{-}\right) / 2$. The hysteresis loop size, $\Delta M_{\text {eff }}$, goes to zero every $60^{\circ}$. We also note that for a broad field range, the absolute amplitudes of $A_{2 \phi}$ and $A_{4 \phi}$ are equal, as shown in the FFT data [Figs. 3(b) and 3(c)]. Under this condition, $\Delta M_{\text {eff }}$ follows the function $f(\phi)=2 A_{2 \phi} \sin \left(\phi-30^{\circ}\right) \cos 3 \phi$, as shown by the solid 
line in Fig. 3(a). This fitting reveals a possible origin of the observed rotational symmetry breaking. The effective superconducting magnetic moment follows the product of $\sin \left(\phi-30^{\circ}\right)$ and $\cos 3 \phi$, rather than the sum of two ordering functions. Therefore, there is a strong coupling between the crystalline symmetry and a nematic ordering in the triangular superconductor $\mathrm{Nb}$-doped $\mathrm{Bi}_{2} \mathrm{Se}_{3}$. The phases between these two sinusoidal functions are locked in the measurement. This suggests that while nematic order is a spontaneous symmetry breaking in the superconducting state, the order seems to be stabilized by the crystalline symmetry. The coupling makes sure that the nematic ordering direction is locked to one of the mirror planes of the triangular lattice. As pointed out in Ref. [13], the nematic order verifies the two-component nature of the superconducting order parameter. Thus, an odd-parity superconducting order is very likely to exist in the ground state, which creates promise for topological superconductivity in $\mathrm{Nb}$-doped $\mathrm{Bi}_{2} \mathrm{Se}_{3}$.

\section{DISCUSSION}

We first discuss the advantages and disadvantages of our approach. As mentioned above, torque magnetometry does not require special instruments, except for an in situ rotator. This method has been used for observing the normal state symmetry breaking of strongly correlated materials $[17,18]$ since it is very sensitive to crystal symmetry breaking. In our study, we show that the amplitude of the hysteresis loop originating from the supercurrent reflects the symmetry of the magnetic susceptibility in the superconducting state. Generally, the hysteresis signal is much larger than the signal from magnetic torque in the superconducting state, so focusing on the symmetry of the hysteresis loop is a good way to detect symmetry breaking in small samples.

There are disadvantages as well in using torque magnetometry to detect symmetry breaking. First, torque magnetometry does not directly measure the superconducting gap symmetry. Thus, even though nematic order is observed by torque, further experiments such as STM are required to understand exactly the origin of the nematicity. Second, since the anisotropy of magnetic susceptibility between in-plane and out-of-plane is one order of magnitude larger than within the plane, a small angle misalignment of the sample could affect the measurement. As shown in Ref. [20], when the sample is misaligned by a few degrees, it shows an extra contribution of the $A_{2 \phi}$ component. Thus, extra attention has to be given to exclude the possibility that $A_{2 \phi}$ is purely from the sample misalignment. However, note that even with the additional contribution, the measurement still reveals two key nematic features: (a) a large $A_{4 \phi}$ component and (b) a vanishing $A_{2 \phi}$ component with high fields.

Next, our study of the magnetic torque demonstrates a symmetric vanishing of the superconducting hysteresis. Magnetic torque is sensitive to the magnetic anisotropy of the superconducting signal, and the hysteretic $M-H$ loops arise from the flux pinning of the vortex solids in the superconducting state. Therefore, the superconducting diamagnetic signal, which comes from the vortex solid, prefers to align along the crystalline axis planes of the triangular lattice. As shown by the crystal structure plot in Fig. 1, the crystalline axis planes exist at every $60^{\circ}$. The diamagnetic signal is maximum when the magnetic field aligns with these preferred directions. The magnetic torque $\vec{\tau}=\mu_{0} \vec{M} \times \vec{H}$ vanishes at these preferred directions when the $M$ vector is exactly collinear (parallel or antiparallel) to the applied field $H$.

A question arises as to why the diamagnetic response is preferred on these particular axes. As pointed out in Ref. [13], the multicomponent superconducting order parameter leads to a nematic superconducting state that spontaneously breaks rotational symmetry of the crystal and exhibits uniaxial anisotropy in the $a b$ plane. As a result, it is expected that the superconducting diamagnetic signal sticks to these preferred axes.

The hysteresis loop is greatly enhanced along one direction. To explore possible explanations for the rotational symmetry breaking, we first consider extrinsic factors that can induce symmetry breaking. First, if this was an electrical or heat transport measurement, it could be from the measurement current or the heat flow itself. However, torque magnetometry is a noncontact measurement, and it does not break the symmetry of the bulk sample. Indeed, this is one of the big advantages of our method, as torque measurement can be easily combined with field rotation. Second, structural phase transition is not likely the origin of the observed rotational symmetry breaking. We observed the smooth heat capacity and resistivity as a function of temperature (see Ref. [20]). The symmetry of the torque measurement in the normal state also shows $\sin 6 \phi$ dependence as expected from the crystalline symmetry, which indicates that the rotational symmetry breaking originates from the superconducting state.

It is also possible that the spontaneous enhanced direction is locked to the strain or structure defects. The magnetization hysteresis loop measures the supercurrent. Thus, the hysteresis loop is determined by the domain structures. The domain pattern might be pinned to some directions by the crystalline defects. However, we note that the effective magnetic susceptibility $\chi$ also shows the rotational symmetry-breaking behavior in the superconducting state. Given that $\chi$ is averaged from the up and down sweeps, based on the Bean model, $\chi$ should not couple to the domain formation. Thus, the rotational symmetry breaking in $\chi$ suggests that the domain structures do not play a key role in rotational symmetry breaking. Furthermore, a periodic vanishing of the hysteresis loop requires unconventional ordering in the superconducting state. Early torque studies on high- $\mathrm{T}_{c}$ cuprates, untwinned 
$\mathrm{YBa}_{2} \mathrm{Cu}_{3} \mathrm{O}_{7}$ [21], and $\mathrm{Tl}_{2} \mathrm{Ba}_{2} \mathrm{CuO}_{6+\delta}$ [22] show in-plane vanishing of the superconducting hysteresis along their crystal axes. In contrast, in classical s-wave superconductors $\mathrm{NbN}$ and $\mathrm{NbSe}_{2}$, the torque signal with $H$ in plane shows no anisotropy [22].

Therefore, we conclude that the symmetry breaking is an intrinsic behavior. There are several possible origins. The first one is odd-parity superconductivity with a nodeless superconducting gap. As shown in Ref. [20], our heat capacity measurement is consistent with a nodeless gap. However, since the superconducting volume is not $100 \%$, there is a remaining density of states which may affect the shape of the heat capacity curve. The second possibility is a nodal even-parity superconducting gap with $E_{g}$ order [13]. Recently, it has been reported that the penetration depth shows a power-law behavior at low temperatures, indicating point nodes [23]. Further experiments such as STM are required to determine whether there is a nodal gap structure in this material.

We would also like to point out a potential difference between our observations in $\mathrm{Nb}$-doped $\mathrm{Bi}_{2} \mathrm{Se}_{3}$ and the similar early experimental observation in $\mathrm{Cu}$-doped $\mathrm{Bi}_{2} \mathrm{Se}_{3}[14,16]$, Sr-doped $\mathrm{Bi}_{2} \mathrm{Se}_{3}$ [15], and the theoretical explanation [13] of nematic order in the spin susceptibility of $\mathrm{Cu}$-doped $\mathrm{Bi}_{2} \mathrm{Se}_{3}$. In the $\mathrm{Cu}$-doped material, quantum oscillations [7,9] and photoemission [6,11] reveal that there is only one bulk Fermi pocket. In contrast, the electronic state in $\mathrm{Nb}$-doped $\mathrm{Bi}_{2} \mathrm{Se}_{3}$ shows at least two distinct Fermi surfaces [24]. Our work calls for further theoretical and experiential exploration of the impact of multi-orbitals in the search for unconventional superconductors. While in Sr-doped $\mathrm{Bi}_{2} \mathrm{Se}_{3}$ large $\mathrm{H}_{c 2}$ anisotropy has been observed between fields parallel and perpendicular to the crystal axis [15], our torque shows a relatively isotropic critical field. This difference could be due to the measurement technology as torque magnetometry is a thermodynamic probe. Further study would be required to elucidate this question.

The unique sensitivity of our in-plane torque magnetometry leads to a promising new tool for probing the superconducting pairing symmetry of other unconventional superconductors. It can elucidate or confirm potential p-wave superconducting symmetry in materials such as $\mathrm{Sr}_{2} \mathrm{RuO}_{4}$ [25] and $\mathrm{UPt}_{3}$ [26,27]. In contrast to those materials, the exponential decay in the heat capacity in $\mathrm{Nb}$-doped $\mathrm{Bi}_{2} \mathrm{Se}_{3}$ suggests a superconducting gap without line nodes. It would be very interesting to investigate whether a nodal superconducting gap would enhance or diminish the nematic order in the superconducting state.

\section{MATERIALS AND METHODS}

We preformed torque magnetometry measurements with our home-built cantilever setup by gluing the $\mathrm{Nb}$-doped $\mathrm{Bi}_{2} \mathrm{Se}_{3}$ single crystal to the end of a thin beryllium copper cantilever. We then placed the cantilever in an external magnetic field $H$. We measured the torque on the cantilever by tracking the capacitance between the metallic cantilever and a fixed gold film underneath, using an AH2700A capacitance bridge with a $7-\mathrm{kHz}$ drive frequency. We calibrated the spring constant of the cantilever by tracking the angular dependence of capacitance caused by the sample weight at zero magnetic field.

The tilt angle $\phi$ is defined as the angle between the direction of the magnetic field and the positive $x$ axis, which is marked in Fig. 1(a) as the direction of the cantilever arm.

The sample heat capacity is measured in the Quantum Design Physical Properties Measurement System using the relaxation method.

The National High Magnetic Field Laboratory provided the magnet and $\mathrm{He} 3$ fridge. During the torque magnetometry measurement, samples were soaked in pumped liquid helium 3, and the magnetic field was swept at $0.25 \mathrm{~T} / \mathrm{min}$.

We performed the magnetization measurement with a Quantum Design Magnetic Properties Measurement System at $H=5$ Oe.

\section{ACKNOWLEDGMENTS}

This work is mainly supported by the Department of Energy (DOE) under Grant No. DE-SC0008110 (magnetization measurement), by the National Science Foundation (NSF) under Grant No. DMR-1255607 (sample growth), and by the David and Lucile Packard Foundation (theory). Supporting measurements were made possible with by the Office of Naval Research through Grant No. N00014-15-12382 (thermodynamic characterization), by the NSF under Grant No. 1307744 (electrical transport characterization), and by the NSF Major Research Instrumentation under Grant No. DMR-1428226 (supports the equipment of the thermodynamic and electrical transport characterizations). Some experiments were performed at the National High Magnetic Field Laboratory (NHMFL), which is supported by NSF Cooperative Agreement No. DMR-084173, by the State of Florida, and by the DOE. We are grateful for the assistance of Tim Murphy, Glover Jones, and Ju-Hyun Park of NHMFL. T. A. thanks the Nakajima Foundation for support. B. J. L. acknowledges support from the National Science Foundation under Grant No. F031543.

[1] M. Z. Hasan and C. L. Kane, Colloquium: Topological Insulators, Rev. Mod. Phys. 82, 3045 (2010).

[2] X. L. Qi and S. C. Zhang, Topological Insulators and Superconductors, Rev. Mod. Phys. 83, 1057 (2011).

[3] Y. S. Hor, A. J. Williams, J. G. Checkelsky, P. Roushan, J. Seo, Q. Xu, H. W. Zandbergen, A. Yazdani, N. P. Ong, and R. J. Cava, Superconductivity in $\mathrm{Cu}_{x} \mathrm{Bi}_{2} \mathrm{Se}_{3}$ and Its Implications for Pairing in the Undoped Topological Insulator, Phys. Rev. Lett. 104, 057001 (2010). 
[4] Zhongheng Liu, Xiong Yao, Jifeng Shao, Ming Zuo, Li Pi, Shun Tan, Changjin Zhang, and Yuheng Zhang, Superconductivity with Topological Surface State in $\mathrm{Sr}_{x} \mathrm{Bi}_{2} \mathrm{Se}_{3}$, J. Am. Chem. Soc. 137, 33 (2015).

[5] L. Fu and E. Berg, Odd-Parity Topological Superconductors: Theory and Application to $\mathrm{Cu}_{x} \mathrm{Bi}_{2} \mathrm{Se}_{3}$, Phys. Rev. Lett. 105, 097001 (2010).

[6] L. A. Wray, S.-Y. Xu, Y. Xia, Y.S. Hor, D. Qian, A. V. Fedorov, H. Lin, A. Bansil, R. J. Cava, and M. Z. Hasan, Observation of Topological Order in a Superconducting Doped Topological Insulator, Nat. Phys. 6, 855 (2010).

[7] B. J. Lawson, G. Li, F. Yu, T. Asaba, C. Tinsman, T. Gao, W. Wang, Y.S. Hor, and Lu Li, Quantum Oscillations in $\mathrm{Cu}_{x} \mathrm{Bi}_{2} \mathrm{Se}_{3}$ in High Magnetic Fields, Phys. Rev. B 90, 195141 (2014).

[8] M. Kriener, K. Segawa, Z. Ren, S. Sasaki, and Y. Ando, Bulk Superconducting Phase with a Full Energy Gap in the Doped Topological Insulator $\mathrm{Cu}_{x} \mathrm{Bi}_{2} \mathrm{Se}_{3}$, Phys. Rev. Lett. 106, 127004 (2011).

[9] B. J. Lawson, Y. S. Hor, and Lu Li, Quantum Oscillations in the Topological Superconductor Candidate $\mathrm{Cu}_{0.25} \mathrm{Bi}_{2} \mathrm{Se}_{3}$, Phys. Rev. Lett. 109, 226406 (2012).

[10] S. Sasaki, M. Kriener, K. Segawa, K. Yada, Y. Tanaka, M. Sato, and Y. Ando, Topological Superconductivity in $\mathrm{Cu}_{x} \mathrm{Bi}_{2} \mathrm{Se}_{3}$, Phys. Rev. Lett. 107, 217001 (2011).

[11] E. Lahoud, E. Maniv, M. S. Petrushevsky, M. Naamneh, A. Ribak, S. Wiedmann, L. Petaccia, Z. Salman, K. B. Chashka, Y. Dagan, and A. Kanigel, Evolution of the Fermi Surface of a Doped Topological Insulator with Carrier Concentration, Phys. Rev. B 88, 195107 (2013).

[12] Y. Qiu, K. Sanders, J. Dai, J. Medvedeva, W. Wu, P. Ghaemi, T. Vojta, and Y.S. Hor, Time Reversal Symmetry Breaking Superconductivity in Topological Materials, arXiv:1512.03519.

[13] L. Fu, Odd-Parity Topological Superconductor with Nematic Order: Application to $\mathrm{Cu}_{x} \mathrm{Bi}_{2} \mathrm{Se}_{3}$, Phys. Rev. B 90, 100509(R) (2014).

[14] K. Matano, M. Kriener, K. Segawa, Y. Ando, and G.-q. Zheng, Spin-Rotation Symmetry Breaking in the Superconducting State of $\mathrm{Cu}_{x} \mathrm{Bi}_{2} \mathrm{Se}_{3}$, Nat. Phys. 12, 852 (2016).

[15] Y. Pan, A. M. Nikitin, G. K. Araizi, Y. K. Huang, Y. Matsushita, T. Naka, and A. de Visser, Rotational Symmetry Breaking in the Topological Superconductor $\mathrm{Sr}_{x} \mathrm{Bi}_{2} \mathrm{Se}_{3}$ Probed by Upper-Critical Field Experiments, Sci. Rep. 6, 28632 (2016).
[16] S. Yonezawa, K. Tajiri, S. Nakata, Y. Nagai, Z. Wang, K. Segawa, Y. Ando, and Y. Maeno, Thermodynamic Evidence for Nematic Superconductivity in $\mathrm{Cu}_{x} \mathrm{Bi}_{2} \mathrm{Se}_{3}$, Nat. Phys., (2016).

[17] S. Kasahara, H. J. Shi, K. Hashimoto, S. Tonegawa, Y. Mizukami, T. Shibauchi, K. Sugimoto, T. Fukuda, T. Terashima, Andriy H. Nevidomskyy, and Y. Matsuda, Electronic Nematicity Above the Structural and Superconducting Transition in $\mathrm{BaFe}_{2}(\mathrm{As} 1-\mathrm{xPx})_{2}$, Nature (London) 486, 382 (2012).

[18] R. Okazaki, T. Shibauchi, H. J. Shi, Y. Haga, T. D. Matsuda, E. Yamamoto, Y. Onuki, H. Ikeda, and Y. Matsuda, Rotational Symmetry Breaking in the Hidden-Order Phase of $\mathrm{URu}_{2} \mathrm{Si}_{2}$, Science 331, 439 (2011).

[19] I. K. Robinson and D. T. Tweet, Surface X-ray Diffraction, Rep. Prog. Phys. 55, 599 (1992).

[20] See Supplemental Material at http://link.aps.org/ supplemental/10.1103/PhysRevX.7.011009 for detailed data of heat capacity, misalignment dependence and sample dependence.

[21] T. Ishida, K. Okuda, H. Asaoka, Y. Kazumata, K. Noda, and H. Takei, Two- and Fourfold ab-Plane Torque Symmetry in Untwinned $\mathrm{YBa}_{2} \mathrm{Cu}_{3} \mathrm{O}_{7}$ Single Crystals, Phys. Rev. B 56, 11897 (1997).

[22] M. Willemin, C. Rossel, J. Hofer, H. Keller, Z. F. Ren, and J.H. Wang, Pairing Symmetry from In-Plane Torque Anisotropy in $\mathrm{Tl}_{2} \mathrm{Ba}_{2} \mathrm{CuO}_{6+\delta}$ Thin Films, Phys. Rev. B 57, 6137 (1998).

[23] M. P. Smylie, H. Claus, U. Welp, W.-K. Kwok, Y. Qiu, Y. S. Hor, and A. Snezhko, Evidence of Nodes in the Order Parameter of the Superconducting Doped Topological Insulator $\mathrm{Nb}_{\mathrm{x}} \mathrm{Bi}_{2} \mathrm{Se}_{3}$ via Penetration Depth Measurements, Phys. Rev. B 94, 180510 (2016).

[24] B. J. Lawson, Paul Corbae, Gang Li, Fan Yu, Tomoya Asaba, Colin Tinsman, Y. Qiu, Y.S. Hor, and Lu Li, Multiple Fermi Surfaces in Superconducting Nb-doped $\mathrm{Bi}_{2} \mathrm{Se}_{3}$, Phys. Rev. B 94, 041114 (2016).

[25] A. P. Mackenzie and Y. Maeno, The Superconductivity of $\mathrm{Sr}_{2} \mathrm{RuO}_{4}$ and the Physics of Spin-Triplet Pairing, Rev. Mod. Phys. 75, 657 (2003).

[26] J. A. Sauls, The Order Parameter for the Superconducting Phases of $\mathrm{UPt}_{3}$, Adv. Phys. 43, 113 (1994).

[27] J. D. Strand, D. J. Bahr, D. J. Van Harlingen, J. P. Davis, W. J. Gannon, and W. P. Halperin, The Transition between Real and Complex Superconducting Order Parameter Phases in $\mathrm{UPt}_{3}$, Science 328, 1368 (2010). 